Z Herz- Thorax- Gefäßchir 2021 · 35:83-96 https://doi.org/10.1007/s00398-021-00424-3 Angenommen: 11. Februar 2021 Online publiziert: 10. März 2021 (c) Springer Medizin Verlag $\mathrm{GmbH}$, ein Teil von Springer Nature 2021

\author{
Dominik Franz ${ }^{1,3} \cdot$ Andreas Wenke $^{1,3} \cdot$ Norbert Roeder ${ }^{2,3}$ \\ 'Franz und Wenke - Beratung im Gesundheitswesen GbR, Münster, Deutschland \\ ${ }^{2}$ Beratung im Gesundheitswesen, Roeder und Partner Ärzte PartG, Senden, Deutschland \\ ${ }^{3}$ DRG-Research-Group, Münster, Deutschland
}

\title{
Abbildung der Herz- und Gefäßchirurgie im aktuellen aG- DRG-System 2021
}

zweiten noch anhaltenden Welle der Pandemie konfrontiert zu werden [1].

Die Akteure in den Kliniken waren im Jahresverlauf zusätzlich mit einer Fülle von Änderungen der gesetzlichen Rahmenbedingungen konfrontiert, deren zeitgerechte Umsetzung und gesetzeskonforme Berücksichtigung berufsgruppenübergreifend alle Mitarbeiterinnen und Mitarbeiter vor große Herausforderungen stellte. Das deutsche DRGInstitut (Institut für das Entgeltsystem im Krankenhaus, InEK) wurde gesetzlich verpflichtet, von den Kliniken zwei unterjährige Lieferungen von Krankenhausroutinedaten anzufordern und auszuwerten. Darüber hinaus wurden wesentliche Regelungen des Reformgesetzes des Medizinischen Dienstes der Krankenversicherung (MDK-Reformgesetz), die ursprünglich in den Jahren 2020 und 2021 umgesetzt werden sollten, ausgesetzt und auf das 2. Halbjahr 2021 oder 2022 verschoben.

\section{》) Die aG-DRG-Systemversion 2021 umfasst v. a. Veränderungen für die Herz- und Gefäßchirurgie}

Trotz dieser erheblichen Beeinträchtigungen liegt die aG-DRG-Systemversion 2021 seit November 2020 vor. Sie umfasst v.a. Veränderungen für die Herzund Gefäßchirurgie, wobei die Thoraxchirurgie - bis auf Bewertungsveränderungen - weitgehend unverändert bleibt [2]. Nachfolgend werden wesentliche aktuelle Veränderungen zu Dokumentation und Kodierung von Diagnosen und Prozeduren sowie zu Struktur und Bewertung des aG-DRG-Systems 2021 für die Herz- und Gefäßchirurgie beschrieben.

\section{Veränderungen auf der Ebene der Diagnosen}

Der ICD-10-GM-Katalog 2021 enthält die neue Diagnose I77.80 zur Kodierung eines penetrierenden Aortenulkus [PAU]. Bisher konnte dieses komplexe Krankheitsbild nur über einen unspezifischen Diagnosekode kodiert werden. Mit der Änderung besteht jetzt Klarheit darüber, dass ein PAU nicht mit dem Effekt einer deutlich höher bewerteten Eingruppierung mit den ICD-Kodes für die Dissektion des betroffenen Aortenabschnitts kodiert werden darf. Darüber hinaus wurde der Diagnoseschlüssel Z99.4 Langzeitige Abhängigkeit vom Kunstherz in die Liste der schweregradrelevanten Nebendiagnosen aufgenommen. Dies bedeutet, dass die Kodierung des ICDKodes Z99.4 als Komorbidität den patientenbezogenen Gesamtschweregrad (PCCL) des Patienten beeinflussen und ggf. in Kombination mit anderen Komorbiditäten $\mathrm{zu}$ einem höheren DRGErlös beitragen kann. „Langzeitig“ bedeutet in diesem Zusammenhang eine Abhängigkeit vom Kunstherz im Umfang von mindestens drei vollen Monaten (- Tab. $1 ;[3,6]$ ). 
Tab. 1 ICD-Veränderungen 2021: zusammenfassende Darstellung der relevanten Veränderungen für Leistungen der Herz- und Gefäßchirurgie

\begin{tabular}{|c|c|c|c|}
\hline Lfd. Nr. & $\begin{array}{l}\text { ICD- } \\
\text { Kode }\end{array}$ & Kurzbezeichnung & Veränderung \\
\hline 1 & 177.80 & $\begin{array}{l}\text { Penetrierendes Aortenulkus } \\
\text { (PAU) }\end{array}$ & Neuer spezifische ICD-Kode \\
\hline 2 & Z99.4 & $\begin{array}{l}\text { Langzeitige Abhängigkeit vom } \\
\text { Kunstherz }\end{array}$ & $\begin{array}{l}\text { Neuaufnahme als schweregradrelevante } \\
\text { Nebendiagnose }\end{array}$ \\
\hline
\end{tabular}

\section{Veränderungen auf der Ebene der Prozeduren}

Für ein Fachgebiet wie die Herz-, Thorax- und Gefäßchirurgie, welches sich ganz überwiegend über chirurgische und interventionelle Eingriffe definiert, sind Veränderungen auf der Ebene einer differenzierten Leistungsabbildung über OPS-Kodes von entscheidender Relevanz. Dies betrifft sowohl die Möglichkeit einer differenzierten Kodierung einerseits als auch einer sach- und leistungsgerechten Erlösfindung auf der Basis unterschiedlicher Ressourcenverbräuche im Rahmen der DRG-Gruppierung andererseits. Der OPS 2021 enthält wichtige Modifikationen für die Herzund Gefäßchirurgie, deren wesentliche Inhalte in $\bullet$ Tab. 2 zusammengefasst sind. Wichtige Umstrukturierungen werden darüber hinaus nachfolgend erläutert [3, 5].

\section{Endovaskuläre Trikuspidal- klappenrekonstruktion}

Der Möglichkeit zur Kodierung einer endovaskulären Trikuspidalklappenrekonstruktion wurde erweitert. Der OPS 2021 sieht nun die Differenzierung einer Trikuspidalklappensegelplastik (535a.50) und einer Trikuspidalklappenanuloplastik (5-35a.51) - jeweils transvenös - vor. Der OPS 5-35a.50 beinhaltet auch die transvenöse Clip-Rekonstruktion der Trikuspidalklappe, wobei die Anzahl der Clips über einen Zusatzkode (5-35a.6 ff) gesondert zu kodieren ist. Im OPS-Kode 5-35a.51 ist auch die Kodierung einer Trikuspidalklappenanuloraphie mit Band enthalten. Ein Erlösunterschied zwischen beiden neuen OPS-Kodes existiert in der aG-DRGVersion 2021 nicht.

Damit bleibt es vorbehaltlich der Entscheidungen des InEK über Anträ- ge zum laufenden Verfahren für neue Untersuchungs- und Behandlungsleistungen (NUB) bei der Situation, dass die Segelplastik an der Mitral- und an der Trikuspidalklappe in die hoch bewertete aG-DRG F98C eingruppiert wird und die Anuloplastik an der Mitralklappe als historisch älteres Verfahren weiterhin in die Basis-DRG F19 ggf. zzgl. NUBEntgelt führt, während das identische Verfahren an der Trikuspidalklappe in die F98C führt. Grundsätzlich ist die Differenzierung zu begrüßen, aufgrund der irreversiblen Zuordnung beider jetzt spezifisch kodierbaren Verfahren zur aG-DRG F98C bleibt die Auswirkung jedoch gering.

\section{Umstrukturierung der Kodierung von Hybridverfahren an der Aorta}

Hybridverfahren an Aorta ascendens, Aortenbogen, Aorta thoracica oder Aorta thoracoabdominalis werden im OPSKatalog 2021 als Zusatzkodes kodiert. Darüber hinaus ist jede verwendete Stent-Prothese gesondert zu kodieren. Die bisherigen OPS-Kodes, die Hybridverfahren an den unterschiedlichen Etagen der Aorta sehr differenziert mit der Zahl der implantierten Stent-Prothesen verbunden haben, wurden aus dem OPS-Katalog gestrichen (• Tab. 3).

Dabei ist die Definition des Hybridverfahrens weiterhin $\mathrm{zu}$ beachten. Ein Hybridverfahren an der Aorta beinhaltet neben der Implantation eines endovaskulär eingebrachten Aorten-Stentgrafts immer auch das Debranching im selben stationären Aufenthalt. Seit dem Jahr 2020 sind dabei nur noch echte Bypass-Eingriffe in Verbindung mit der Implantation aortaler Stentgrafts als Hybrideingriffe klassifiziert, während die Kombination mit Gefäßtranspositionen nicht mehr als Hybrideingriffe gelten.
Der Umbau der OPS-Kodes für die Hybridverfahren in nicht weiter differenzierte Zusatzkodes ist insofern zu begrüßen, als damit die bisherige redundante Erfassung der Zahl der Stentgrafts entfällt. Auch als Zusatzkodes lösen die entdifferenzierten OPS-Kodes auch weiterhin das entsprechende Zusatzentgelt aus, sodass auf der Ebene der Vergütung keine Änderungen zu erwarten sind.

\section{Zusammenfassung von Zusatzinformationen zu Operationen an Blutgefäßen}

Zusatzinformationen zu Operationen an Blutgefäßen werden im OPS 2021 unter 5-39a- zusammengefasst. Dies betrifft Umstrukturierungen für bereits kodierbare Verfahren wie die intraoperative Anwendung eines Embolieprotektionssystems (ehemals 5-399.e - nun 5-39a.0), die temporäre atraumatische Okklusion von Blutgefäßen mit viskösem Polymer mit Umkehrphase (ehemals 5-399.g nun 5-39a.1), die Anwendung einer Gefäßprothese mit integriertem Stent (ehemals 5-399.h - nun 5-39a.2) und das Zusammenfügen eines Venenbypass-Grafts aus mindestens zwei Teilstücken (ehemals 5-399.j - nun 5-39a.3).

\section{》) Der Zusatz-Kode 5-39a.4 Intraoperativ angefertigte Gefäßprothese wurde neu implementiert}

Erweitert wird diese Liste durch den neu implementierten Zusatz-Kode 5-39a.4 Intraoperativ angefertigte Gefäßprothese. Hierdurch wird die Verwendung einer durch Zusammennähen von Patches oder Rohrprothesen intraoperativ angefertigten Gefäßprothese kodierbar.

\section{Trennung in "patientenindividuell hergestellte" und "intraoperativ angefertigte" Gefäßprothese}

Eine Unschärfe bestand bisher bezüglich der Kodierung intraoperativ angefertigter oder modifizierter Gefäßprothesen. Dabei waren sowohl komplett intraoperativ z. B. im Rahmen von Infekt- 
Z Herz- Thorax- Gefäßchir 2021 · 35:83-96 https://doi.org/10.1007/s00398-021-00424-3

(c) Springer Medizin Verlag GmbH, ein Teil von Springer Nature 2021

\section{Franz $\cdot$ A. Wenke $\cdot$ N. Roeder}

\section{Abbildung der Herz- und Gefäßchirurgie im aktuellen aG-DRG-System 2021}

\section{Zusammenfassung}

Einleitung. Das Jahr 2020 wird immer im Schatten der durch die "coronavirus disease 2019" (COVID-19) ausgelösten Pandemie stehen. Die ersten 3 Quartale 2020 waren geprägt von einem beispiellosen Herunterfahren der elektiven stationären Leistungserbringung und einem Freihalten von Intensivkapazitäten für die Behandlung von COVID-19-Patienten. Auch für die Herzchirurgie resultierten dadurch deutliche Auswirkungen auf die Leistungserbringung. Die Akteure in den Kliniken waren im Jahresverlauf zusätzlich mit einer Fülle von Änderungen der gesetzlichen Rahmenbedingungen konfrontiert. Dennoch wurde das aG-DRG-System 2021 durch das Institut für das Entgeltsystem im Krankenhaus (InEK) kalkuliert. Dieser Beitrag beschreibt und bewertet die wesentlichen Veränderungen des aG-DRG-Systems 2021 für die HerzThorax- und Gefäßchirurgie.
Methodik. Analyse der relevanten Diagnosen, Prozeduren und G-DRG-Strukturen in den Systemversionen 2020 und 2021 anhand der durch InEK und das Bundesinsitut für Arzneimittel und Medizinprodukte (BfArM) publizierten Informationen.

Ergebnisse. Erweiterungen der relevanten Klassifikationssysteme für Diagnosen (ICD-10GM 2021) und Prozeduren (OPS 2021) führen zu einer Zunahme spezifischerer Kodierungen von wesentlichen Interventionen und Operationen der Herz- und Gefäßchirurgie. Im Rahmen der Anpassung der G-DRGStrukturen sind besonders die Kondensation der bisherigen Fallpauschalen für die Herztransplantation zur aG-DRG A05Z und Abwertungen koronarer Bypassoperationen und rekonstruktiver Gefäßeingriffe zu nennen. Schlussfolgerung. Für die Herz- und Gefäßchirurgie finden sich vielfältige Verän- derungen mit z. T. erheblichen Auswirkungen auf die Fallerlöse. Hinzu kommt, dass die Auswirkungen der "Coronapandemie" für viele deutsche Kliniken noch nicht abschließend absehbar sind. Ein weiterer, immer dringlicherer insbesondere die Gefäßmedizin betreffende Einflussfaktor ist der zunehmende Druck zur Ambulantisierung von Leistungen. Hier wird der im Jahre 2022 erwartete und voraussichtlich deutlich erweiterte Katalog für Ambulantes Operieren im Krankenhaus (AOP-Katalog) den Zwang zur ambulanten Erbringung bisher noch stationär erbrachter Leistungen sicher noch einmal deutlich erhöhen.

Schlüsselwörter

Thoraxchirurgie - Finanzierung des Gesundheitswesens · Internationale Klassifikation der Krankheiten $\cdot$ Kodierung $\cdot$ Medizinökonomie

\section{Depiction of cardiovascular surgery in the current modified German DRG system 2021}

\section{Abstract}

Introduction. The year 2020 will always stand in the shadow of the pandemic triggered by the coronavirus 2019 (COVID-19). The first three quarter years of 2020 were characterized by an unprecedented reduction of elective inpatient services and a prioritization of intensive care capacity for the treatment of COVID-19 patients. This also resulted in clear consequences for the services provided in cardiac surgery. In addition, during the course of the year the personnel in hospitals were confronted with a plethora of amendments in the legal framework conditions. Nevertheless, the modified German diagnosis-related groups (G-DRG) system 2021 was calculated by the Institute for the Remuneration System in Hospitals (InEK). This article describes and assesses the most important amendments of the modified G-DRG system 2021 for cardiac, thoracic and vascular surgery.
Methods. Analysis of the relevant diagnoses, procedures and G-DRG structures in the system versions for 2020 and 2021 based on the information published by the InEK and the German Federal Institute for Drugs and Medical Devices (BfArM).

Results. Expansions of the relevant classification systems for diagnoses (ICD-10-GM 2021) and procedures (OPS 2021) lead to an increase in specific coding of essential interventions and operations in cardiovascular surgery. Within the framework of the adaptation of the G-DRG structures, the condensation of the previous fixed rates for heart transplantation to G-DRG A05Z and devaluation of coronary bypass operations and reconstructive vascular interventions are particularly important. Conclusion. For cardiovascular surgery there are manifold amendments with sometimes substantial repercussions for the case proceeds. Additionally, for many German hospitals the effects of the corona pandemic are not yet finally foreseeable. A further increasingly more urgent influencing factor particularly affecting vascular medicine is the increasing pressure to promote outpatient treatment. In this respect, the catalogue for outpatient operations in hospitals (AOP), which is expected in 2022 and will presumably be much expanded, will once again clearly increase the enforcement of outpatient performance of services that were previously performed as inpatient treatment.

Keywords

Thoracic surgery - Healthcare financing . International classification of diseases . Coding $\cdot$ Health economics 
Tab. 2 OPS-Veränderungen 2021: zusammenfassende Darstellung der relevanten Veränderungen für Leistungen der Herz- und Gefäßchirurgie

Lfd. Nr. OPS 2021 Kurzbezeichnung Veränderung

\begin{tabular}{|c|c|c|}
\hline \multirow[t]{3}{*}{1} & 5-351.- & Ersatz von Herzklappen durch Prothese \\
\hline & 5-352.- & Wechsel von Herzklappenprothesen \\
\hline & 5-358.- & Operationen bei kongenitalen Klappenanomalien des Herzens \\
\hline \multirow[t]{2}{*}{2} & 5-357.- & Operationen bei kongenitalen Gefäßanomalien \\
\hline & 5-359.- & Andere Operationen am Herzen bei kongenitalen Anomalien \\
\hline 3 & 5-35.a7 & $\begin{array}{l}\text { Minimal-invasive Operationen an Herzklappen: Verschluss einer paraval- } \\
\text { vulären Leckage, transapikal }\end{array}$ \\
\hline
\end{tabular}

Neuer Hinweis bzgl. der Mitimplantation eines klappentragenden Gefäßstückes bei Verwendung eines dezellularisierten Allotransplantates

Neuer Hinweis bzgl. der gesonderten Kodierung eines dezellularisierten Allotransplantates

8-83d.7 Verschluss einer paravalvulären Leckage durch perkutan-transluminale Gefäßintervention

5-35a.5- Endovaskuläre Trikuspidalklappenrekonstruktion

5 5-38a.a Bei Hybridverfahren an Aorta ascendens, Aortenbogen oder Aorta thoracica

5-38a.b Bei Hybridverfahren an der Aorta thoracoabdominalis

5-380.9m Inzision, Embolektomie und Thrombektomie von Blutgefäßen: Tiefe Venen: Gefäßprothese

Neues Exklusivum, dass sich auf den jeweils anderen der beiden OPS-Kodes bezieht

Neue OPS-Kodes zur Differenzierung in Segelplastik und Anuloplastik, transvenös

Umwandlung in Zusatzkodes, Hinweis auf die gesonderte Kodierung verwendeter Stent-Prothesen Löschen der mit diesen Kodes bisher redundant zur sonstigen Kodierung anzugebenden Anzahl von aortalen Stent-Prothesen

Neue OPS-Kodes

5-380.a7 Inzision, Embolektomie und Thrombektomie von Blutgefäßen: Oberflächliche Venen: Gefäßprothese

5-383.44 Resektion und Ersatz (Interposition) von (Teilen von) Blutgefäßen: Arteri- Neuer OPS-Kode en thorakal: Truncus pulmonalis

8 5-393.39 Anlegen eines anderen Shunts und Bypasses an Blutgefäßen: Aorta: Aortomesenterial

Neue OPS-Kodes

5-393.3a Anlegen eines anderen Shunts und Bypasses an Blutgefäßen: Aorta: Aortorenal

5-394.1- Revision einer Blutgefäßoperation: Revision einer Anastomose: Implantat

5-394.3- ... Wechsel eines vaskulären Implantates...

5-394.8 Revision eines vaskulären Transplantates

5-394.9- ... Wechsel eines vaskulären Transplantates...

5-394.a Entfernung eines vaskulären Transplantates

10 5-39a.0 Intraoperative Anwendung eines Embolieprotektionssystems

5-39a.1 Temporäre atraumatische Okklusion von Blutgefäßen mit viskösem Polymer mit Umkehrphase

Neustrukturierung bereits bestehender OPS-Kodes (alte Inhalte und OPS-Texte mit neuen Schlüsselnummern)

5-39a.2 Anwendung Gefäßprothese mit integriertem Stent

5-39a.3 Zusammenfügen eines Venenbypass-Grafts aus mindestens zwei Teilstücken

11 5-39a.4 Zusatzinformationen zu Operationen an Blutgefäßen: Intraoperativ angefertigte Gefäßprothese

12 8-83c.g- $\begin{aligned} & \text { Andere (perkutan-)transluminale Gefäßintervention: endovaskuläre An- } \\ & \text { lage einer AV-Fistel durch Einlage von Stent-Grafts zur Arterialisierung }\end{aligned}$

Neuer OPS-Kode tiefer Venen

situationen aus mit bovinen Patches erzeugte Prothesen als auch das Schaffen zusätzlicher Öffnungen in industriell vorgefertigten Produkten betroffen. Insbesondere vor dem Hintergrund der wenig befriedigenden und für das 2021 wieder beseitigten Situation der Auslösung des Zusatzentgeltes 2020-53 für den Zusatz-OPS-Kode 5-38a.w Endovaskuläre Implantation von Stent-Prothesen: Patientenindividuell hergestellte Stent-
Prothesen bestand hier ein erheblicher Anreiz, auch diese intraoperativ angefertigten Prothesen mit diesem OPSKode zu verschlüsseln. Mit dem neuen OPS-Kode 5-39a.4 Zusatzinformationen zu Operationen an Blutgefäßen: Intraoperativ angefertigte Gefäßprothese (s.oben) und dem zugehörigen Exklusivum „modifizierte konfektionierte Stent-Prothesen“ ist hier Klarheit geschaffen worden (• Tab. 7).
Differenzierung der OPS-

Kodierung von Revisionen von Gefäßoperationen

Zwischen Leistungserbringern und den Medizinischen Diensten gab es bei der Verwendung von OPS-Kodes für Revisionen von Gefäßoperationen unterschiedliche Auffassungen bezüglich des Begriffs „Implantat“. Beispielsweise handelt es sich bei einem Venenbypass 


\begin{tabular}{|c|c|c|c|c|}
\hline OPS 2020 & OPS-Text 2020 & & OPS 2021 & OPS-Text 2021 \\
\hline $5-38 a . a 0$ & \multirow{3}{*}{$\begin{array}{l}\text { Bei Hybridverfahren an } \\
\text { Aorta ascendens, Aor- } \\
\text { tenbogen oder Aorta } \\
\text { thoracica }\end{array}$} & Mit Implantation einer Stent-Prothese & \multirow[t]{3}{*}{$5-38 a . a$} & \multirow{3}{*}{$\begin{array}{l}\text { Bei Hybridverfahren an Aorta ascendens, } \\
\text { Aortenbogen oder Aorta thoracica } \\
\text { Hinw.: Dieser Kode ist ein Zusatzkode. Jede } \\
\text { verwendete Stent-Prothese ist gesondert zu } \\
\text { kodieren }\end{array}$} \\
\hline $5-38 a . a 1$ & & Mit Implantation von zwei Stent-Prothesen & & \\
\hline $5-38 a . a 2$ & & $\begin{array}{l}\text { Mit Implantation von drei oder mehr Stent- } \\
\text { Prothesen }\end{array}$ & & \\
\hline $5-38 a . b 0$ & \multirow{3}{*}{$\begin{array}{l}\text { Bei Hybridverfahren an } \\
\text { der Aorta thoracoabdomi- } \\
\text { nalis }\end{array}$} & Mit Implantation einer Stent-Prothese & \multirow[t]{3}{*}{$5-38 a \cdot b$} & \multirow{3}{*}{$\begin{array}{l}\text { Bei Hybridverfahren an der Aorta thoracoab- } \\
\text { dominalis } \\
\text { Hinw.: Dieser Kode ist ein Zusatzkode. Jede } \\
\text { verwendete Stent-Prothese ist gesondert zu } \\
\text { kodieren }\end{array}$} \\
\hline $5-38 a . b 1$ & & Mit Implantation von zwei Stent-Prothesen & & \\
\hline $5-38 a . b 2$ & & $\begin{array}{l}\text { Mit Implantation von drei oder mehr Stent- } \\
\text { Prothesen }\end{array}$ & & \\
\hline
\end{tabular}

um ein Transplantat und nicht um ein Implantat. Der OPS 2021 wurde zur Klarstellung um Differenzierungen von Revisionen von Gefäßoperationen erweitert. Hierbei wird eine spezifische Kodierung der Revision eines Implantates, eines Transplantates sowie eines arteriovenösen Shunts ermöglicht (OPS 5-934.1-). Eine weitere Differenzierung bezüglich Implantat und Transplantat erfolgt nun auch für die bisherigen OPSKodes 5-394.3 Wechsel eines vaskulären Implantates und 5-394.x Revision einer Gefäßoperation (• Abb. 1).

Vor dem Hintergrund der noch darzustellenden Abwertung (s.unten) der meisten dieser jetzt neu differenzierten OPS-Kodes im aG-DRG-System 2021 reduziert sich die Bedeutung der neuen Gliederung der Revisionseingriffe weitgehend, wobei diese Abwertung zum Zeitpunkt der OPS-Antragstellung durch die Deutsche Gesellschaft für Gefäßchirurgie noch nicht bekannt war.

\section{Deutsche Kodierregeln und Schlichtungsausschuss nach $\S 19$ KHG}

Das MDK-Reformgesetz regelte die Einführung eines neuen Schlichtungsausschusses auf Bundesebene zur Klärung strittiger Kodier- und Abrechnungsfragen (Schlichtungsausschuss nach $\$ 19$ KHG). Der neue Schlichtungsausschuss wurde mit der Klärung von 69 Kodierfragen beauftragt, die zwischen der Sozialmedizinischen Expertengruppe 4 (Vergütung und Abrechnung) der Medizinischen Dienste und dem Fachausschuss für ordnungsgemäße Kodierung und Abrechnung der Deutschen Gesellschaft für Medizincontrolling bis
Ende 2019 als strittig festgestellt worden waren. Die Entscheidungen des Schlichtungsausschusses gelten als Kodierregeln und werden in einem Anhang zu den Deutschen Kodierrichtlinien aufgeführt [4].

Der Beginn der Gültigkeit der jeweiligen Entscheidungen richtet sich nach dem Zeitpunkt ihrer Veröffentlichung. Für die Herz-, Thorax- und Gefäßchirurgie sind neun Entscheidungen des Schlichtungsausschusses von Bedeutung und in $\bullet$ Tab. 4 zusammengefasst.

Seit Jahresanfang 2021 können auch Krankenkassen, Krankenhäuser, das BMG und von „mit Kodierung von Krankenhausleistungen befasste" Fachgesellschaften den neuen Schlichtungsausschuss zur Klärung weiterer strittiger Kodier- und Abrechnungsfragen anrufen. Hierzu wurde ein formales Antragsverfahren etabliert. Für weitere Hintergrundinformationen sei auf die Homepage des InEK unter https://www. g-drg.de/Schlichtungsausschuss_nach_ 19_KHG verwiesen.

\section{Entwicklungen für die Herz- und Gefäßchirurgie auf der Ebene der aG-DRG-Struktur}

Die Anzahl der bundeseinheitlich bewerteten aG-DRG innerhalb der Hauptdiagnosekategorie (MDC) 05 Krankheiten und Störungen des Kreislaufsystems beträgt in der aG-DRG-Version 2021 143, 4 weniger als im Vorjahr. Wesentliche Umstrukturierungen gibt es für 2021 hinsichtlich der Zusammenfassung der aGDRG A05Z Herztransplantation sowie hinsichtlich der Bewertung von Revisionseingriffen und von peripheren StentInterventionen. Dies betrifft vor allen die beiden Basis-DRG F14 Komplexe und mehrfache Gefäßeingriffe und F59 Mäßig komplexe Gefäßeingriffe. In - Tab. 5 sind die wichtigsten Veränderungen innerhalb der aG-DRG-Struktur der MDC 05 , fokussiert für die Leistungsbereiche der Herz- und Gefäßchirurgie, zusammenfassend dargestellt. Die nachfolgenden Darstellungen von G-DRG-Bewertungen beziehen sich auf Normallieger, also Fallkonstellationen mit einer stationären Verweildauer zwischen der oberen und der unteren Grenzverweildauer der jeweiligen Fallpauschale und wurden mit dem Bundesbasisfallwert 2020 in Höhe von $3679,62 €$ berechnet, da der Bundesbasisfallwert 2021 bei der Erstellung dieses Beitrages noch nicht veröffentlicht war.

\section{Zusammenfassung von Herztransplantationen in der DRG A05Z}

In der G-DRG-Systemversion 2020 wurden Fallkonstellationen mit einer Herztransplantation in Abhängigkeit von der Beatmungsdauer und der Zahl intensivmedizinischer Komplexpunkte in die beiden G-DRGs A05A oder A05B eingruppiert. Diese Differenzierung basierte gemäß der vom InEK publizierten Datengrundlage (Stand 2018) auf einer Fallzahl von insgesamt 54 Normalliegern [8]. Für die Kalkulation der G-DRG-Version 2021 standen dem InEK insgesamt nur 17 Normallieger zur Verfügung. Aufgrund dieser geringen Fallzahl wurden die G-DRG A05A und A05B der Vorjahresversion zur G-DRG A05Z der G-DRG-Version 2021 zusammengefasst [7].

Diese Änderung führt formal für die diese Leistung erbringenden Kliniken in 

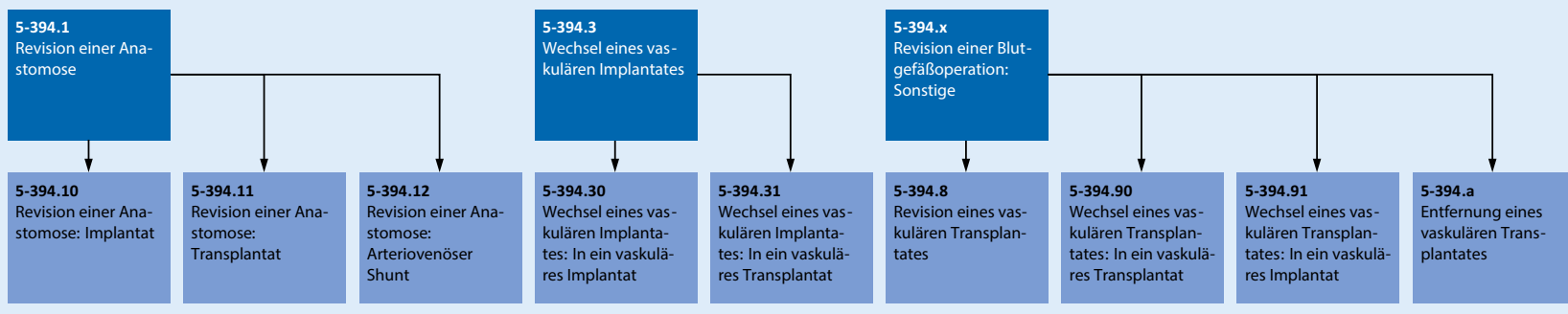

Abb. $1 \Delta$ Differenzierung der OPS-Kodierung von Revisionen von Gefäßoperationen

die Notwendigkeit, diese hochkomplexen Leistungen aufder Basis oftmals nicht oder eben auch nur unzureichend existierender Kostendaten des eigenen Hauses für die Entgeltverhandlungen zu kalkulieren. Inwieweit hier die Vergütung des Vorjahreskataloges als Basis herangezogen werden kann, bleibt abzuwarten.

\section{Bewertungsveränderung des \\ Aortenklappenersatzes in Kombination mit Mehrfachvenen- bypässen}

Die operative Therapie einer schweren Aortenklappeninsuffizienz erfolgt durch den Ersatz der insuffizienten Klappe. Hierfür stehen verschiedene Herzklappenprothesen zur Verfügung. Der Herzklappenersatz erfolgt im klinischen Alltag allein oder in Kombination mit anderen operativen Eingriffen, in der Regel z. B. der Anlage eines bzw. mehrerer arterieller und/oder venöser Bypässe. Die DRG-Zuordnung von Fallkonstellationen mit unterschiedlichen Arten des Klappenersatzes in Abhängigkeit von der Kombination mit einem ein-, 2- oder 3-fachen aortokoronaren Bypass mit autogenen Arterien oder Venen zeigt - Abb. 2. Hinsichtlich des Klappenersatzes erfolgt der Vergleich mit einer Bioprothese (5-351.02), einem nahtfreien selbstexpandierenden Xenotransplantat (5-351.05) und einem ballonexpandierendem Xenotransplantat mit Fixierungsnähten. Fallkonstellationen mit einem Aortenklappenersatz mit einem ballonexpandierendem Xenotransplantat mit Fixierungsnähten in Kombination mit einem 2-fachen Venenbypass oder einem 3-fachen Bypass mit Venen oder Arterien werden hierbei in der aG-DRG-Systemversion
2021 der G-DRG F03B Herzklappeneingriff mit Herz-Lungen-Maschine, mit Mehrfacheingriff oder Alter $<1$ Jahr oder Eingriff in tiefer Hypothermie oder IntK >392/368/- Aufwandspunkte oder bestimmter anderer komplizierender Konstellation oder pulmonale Endarteriektomie zugeordnet. Dies bedeutet einen Rückgang der DRG-Bewertung im Vergleich zur Vorjahresversion.

Mit dieser Anpassung des aG-DRGSystems 2021 setzt sich die bereits in den Vorjahren zu beobachtende Angleichungstendenz der nahtfixierten Herzklappen im Vergleich zu den nahtfreien Klappen fort, wobei auch weiterhin teilweise eine höhere Vergütung der nahtfixierten Klappen gegenüber den nahtfreien und insbesondere natürlich den „konventionellen" Herzklappenprothesen im Kombinationseingriff besteht.

\section{Bereinigung der Basis-DRG F07}

Mit dem Systemjahr 2021 wurde die Basis-DRG F07 Andere Eingriffe mit HerzLungen-Maschine um viele bisher gruppierungsrelevante Zusatzeingriffe bereinigt. In der Praxis werden dabei die nachfolgend beispielhaft aufgeführten weiterhin kodierbaren, jetzt aber nicht mehr für die F07 gruppierungsrelevanten Leistungen besonders bedeutsam sein:

- Abwertung OPS 5-379.5 Reoperation an Herz und Perikard,

- Abwertung OPS 5-370.1 Perikardiotomie und Kardiotomie: Perikardiotomie,

- Abwertung Desobliteration der Koronararterien (OPS 5-360 ff.).

Die dargestellten und in geringerer Fallzahl auch noch weitere Detailabwertungen führen je nach Leistungsspektrum der Klinik zu z.T. erheblichen Verlusten an Erlösen im Vorjahresvergleich insbesondere die Abwertung der aufwendigeren Reoperationen.

Neben den beschriebenen Abwertungen findet sich eine für manche herzchirurgische Kliniken allerdings ebenfalls bedeutsame Aufwertung innerhalb der Basis-DRG F07. Aufgewertet wird dabei der OPS-Kode 5-384.12 Resektion und Ersatz (Interposition) an der Aorta: Aorta ascendens mit Reimplantation der Koronararterien: Mit Rohrprothese bei Aneurysma. Dieser führt in 2021 neu in die aG-DRG F07A Andere Eingriffe mit Herz-Lungen-Maschine, Alter $<1 \mathrm{Jahr}$ oder mit best. kompliz. Konstellation oder komp. Operation oder IntK >-/368 /- P. oder Alter $<18$ Jahre mit Reop. Herz od. Perikard oder and. kompliz. Konstellation, mit best. kompl. Eingriffen, wobei im Beispiel der $\bullet$ Abb. 3 der OPS-Kode für die Reoperation weiterhin erhebliche Erlösbedeutung besitzt und nicht von der beschriebenen Abwertung betroffen ist.

\section{Abwertung von Revisionseingrif- fen an Blutgefäßen}

Revisionseingriffe an Blutgefäßen waren bis zur aG-DRG-Systemversion 2020 ein Attribut komplexer Gefäßeingriffe in den Basis-DRG F08 Rekonstruktive Gefäßeingriffe und F14 Komplexe oder mehrfache Gefäßeingriffe außer große rekonstruktive Eingriffe. Während in der Basis-DRG F08 bereits vor einigen Jahren der OPS-Kode 5-394.1 Revision einer Blutgefäßoperation: Revision einer Anastomose abgewertet worden war, hatte dort der OPS-Kode 5-394.2 Revision einer Blutgefäßoperation: Revision eines vaskulären Implantates 


\section{Lfd. Nr. Schlich- Kurzbezeichnung tungs-ID \\ 1 KDE 533 Vitien mehrerer Herzklappen gleichzeitig}

KDE 174 Operativer äußerer Kreislauf (bei Anwendung der Herz-LungenMaschine) mit tiefer Hypothermie

4 KDE 338 Tachyarrhythmia absoluta aufgrund von Vorhofflimmern

KDE 585 Kodierung eines erst intraoperativ entdeckten angeborenen Herzfehlers bei Erwachsenen ohne Änderung des geplanten Operationsablaufs

KDE 558 Anlage einer extrakorporalen Membranoxygenierung (ECMO)/ eines Extracorporeal Life Support System (ECLS) gehört zu den speziellen Interventionen auf der Intensivstation

KDE 466 Kodierung Hauptdiagnose bei Auf(ex 20) nahme ausschließlich zur Thrombektomie bei Thrombose eines AVShunts

KDE 433 Kodierung des ICD-Kodes U55.Erfolgte Registrierung zur Organtransplantation
Entscheidung

Liegen gleichzeitig Vitien mehrerer Herzklappen vor, so ist für jede betroffene Herzklappe jeweils der Kode, welcher Art und Ursache des Vitiums zutreffend beschreibt, zu kodieren Kombinations-OPS nur dann verwenden, wenn die genannten Informationen klappenspezifisch genau beschrieben werden

Die manuelle Herzkompression zur Reanimation bei bereits im Rahmen einer anderen Operation eröffnetem Thorax ist mit dem Kode 5-379.0 Andere Operationen an Herz und Perikard, Offene Herzmassage zu kodieren

Der OPS-Kode 8-851.4 Operativer äußerer Kreislauf (bei Anwendung der Herz-Lungen-Maschine) mit tiefer Hypothermie ist nur bei Vorliegen einer Temperatur innerhalb des dort genannten Temperaturintervalls (zum Zeitpunkt der Schlichtung, Temperatur von 20 bis unter $26^{\circ} \mathrm{C}$ ) zu kodieren

Eine Tachyarrhythmia absoluta aufgrund von Vorhofflimmern ist mit dem zutreffenden Kode aus 148.- Vorhofflimmern und Vorhofflattern zu kodieren. Der Kode 147.1 Supraventrikuläre Tachykardie ist für die Tachyarrhythmia absoluta bei Vorhofflimmern nicht zusätzlich zu kodieren

Auch der Stent, dessen Einlage nicht gelingt und der daraufhin verworfen wird, wird bei der Kodierung der Zahl der Stents berücksichtigt

Bei einem erwachsenen Patienten, der zur geplanten Operation einer Aortenklappenstenose kommt und erst intraoperativ eine bikuspidale Aortenklappe mit Stenose festgestellt wird, deren Vorhandensein nicht zu einer Änderung im geplanten Operationsablauf führt, ist der Kode I35.0 Aortenklappenstenose als Hauptdiagnose zu kodieren. Die bikuspidale Aortenklappe wird in diesem Fall nicht mit einem Kode aus Q23.- Angeborene Fehlbildungen der Aorten- und der Mitralklappe als Nebendiagnose kodiert

Die ECMO/das ECLS gehört zu den speziellen Interventionen auf der Intensivstation. Im Rahmen einer intensivmedizinischen Komplexbehandlung ist nur die Anlage der ECMO/des ECLS bei der Ermittlung der diesbezüglichen Aufwandspunkte für den TISS zu berücksichtigen

Wird ein Patient mit dialysepflichtiger Nierenkrankheit ausschließlich zur Thrombektomie bei Thrombose eines AV-Shunts (Shunt aus körpereigenem Material) elektiv aufgenommen, so ist für die Thrombose des AV-Shunts der Kode T82.8 Sonstige näher bezeichnete Komplikationen durch Prothesen, Implantate oder Transplantate im Herzen und in den Gefäßen als Hauptdiagnose zu kodieren

Zeitpunkt der Kodierung des ICD-Kodes U55.- Erfolgte Registrierung zur Organtransplantation. Diese Registrierung muss nicht im aktuellen stationären Aufenthalt erfolgt sein. Der Kode ist zu verwenden, wenn die erfolgte Registrierung weiterhin Gültigkeit besitzt weiter eine den erlössteigernde Wirkung (• Abb. 4).

Mit dem aG-DRG-System 2021 wurden sowohl der noch verbliebene OPSKode für die Revision eines vaskulären Implantates aus der Basis-DRG F08 als auch beide im Vorjahr noch gruppierungsrelevanten OPS-Kodes für die Revision eines vaskulären Implantates und für die Revision einer Anastomose aus der Basis-DRG F14 gestrichen.

Fallkonstellationen ohne rekonstruktive Gefäßeingriffe wurden bisher im Zusammenhang mit den dargestellten OPS-Kodes für Revisionseingriffe unabhängig vom Stadium der peripheren arteriellen Verschlusskrankheit (pAVK) der Basis-DRG F14 Komplexe und mehr- fache Gefäßeingriffe zugeordnet. Die dargestellten Veränderungen der aG-DRGSystemversion 2021 heben diese Gruppierungsrelevanz für die beiden OPSKodes 5-394.1 Revision einer Anastomose und 5-394.2 Revision eines vaskulären Implantates in Verbindung mit weniger komplexen Stadien der pAVK auf (- Tab. 6), wodurch eine erhebliche Erlösreduktion einer nichtunerheblichen Anzahl von Fallkonstellationen resultiert (- Abb. 5). Die in - Tab. 2 abgebildeten OPS-Kodes sind für die OPS-Version 2021 z. T. differenziert worden (s. oben). Inwieweit sich durch die spezifische Kodierung von Implantaten und Transplantaten ggf. weitere Veränderungen der Zuordnung zu DRG-Fallpauschalen ergeben, wird frühestens in der aGDRG-Version 2023 durch das InEK kalkulierbar sein.

Gerade in der Gefäßchirurgie sind Revisionseingriffe häufig. Hierbei handelt es sich in der Regel nicht um Frührevisionen im Rahmen der unmittelbar postoperativen Komplikationsbehandlung, sondern zumeist um Revisionen früherer Eingriffe aus anderen Aufenthalten, welche z.T. auch nicht in der eigenen Klinik oder Abteilung durchgeführt wurden.

Die grundsätzliche Unklarheit bezüglich der Kodierung, welche regelhaft in Auseinandersetzungen zwischen Kliniken und Kostenträgern mündete, bleibt bestehen. Es ist jedoch zu erwarten, dass es zu einer Reduktion von Auseinander- 


\section{Krankenhausmanagement}

\begin{tabular}{|c|c|c|c|c|}
\hline Nr. & DRG 2020 & DRG 2021 & $\begin{array}{l}\text { DRG-Bezeichnung } 2021 \text { oder Kurz- } \\
\text { bezeichnung }\end{array}$ & Veränderung \\
\hline 1 & A05A/B & A05Z & Herztransplantation & Kondensation der bisher zweifach differenzierten DRG \\
\hline 2 & F03A & F03B & $\begin{array}{l}\text { Herzklappeneingriff mit Herz-Lungen- } \\
\text { Maschine... }\end{array}$ & $\begin{array}{l}\text { Abwertung Aortenklappenersatz durch ballonexpandierendes Xenotrans- } \\
\text { plantat mit Fixierungsnähten in Kombination mit Mehrfachvenenbypässen }\end{array}$ \\
\hline 3 & F07C & F07B & $\begin{array}{l}\text { Andere Eingriffe mit Herz-Lungen- } \\
\text { Maschine ... }\end{array}$ & Aufwertung der Ross-Operation (5-354.0c) \\
\hline 4 & F09C & F09B & $\begin{array}{l}\text { Andere kardiothorakale Eingriffe ohne } \\
\text { Herz-Lungen-Maschine }\end{array}$ & Aufwertung epikardialer Ablationen \\
\hline 5 & F07C & F06E & Koronare Bypass-Operation... & $\begin{array}{l}\text { Abwertung der Desobliteration der Koronargefäße im Zusammenhang mit } \\
\text { Bypass-Anlage }\end{array}$ \\
\hline 6 & F08B & F08C/D & Rekonstruktive Gefäßeingriffe ... & Abwertung des OPS-Kodes 5-394.2 Revision eines vaskulären Implantates \\
\hline 7 & F08D & F08F & Rekonstruktive Gefäßeingriffe ... & Abwertung von Mehretageneingriffen \\
\hline \multirow[t]{2}{*}{8} & \multirow[t]{2}{*}{$\begin{array}{l}\text { Basis-DRG } \\
\text { F14 }\end{array}$} & \multirow[t]{2}{*}{$\begin{array}{l}\text { Basis-DRG } \\
\text { F59 }\end{array}$} & \multirow[t]{2}{*}{ Mäßig komplexe Gefäßeingriffe ... } & $\begin{array}{l}\text { Abwertung der OPS-Kodes 5-394.1 Revision einer Anastomose und 5-394.2 } \\
\text { Revision eines vaskulären Implantates }\end{array}$ \\
\hline & & & & Abwertung verschiedener Stent-Kombinationseingriffe \\
\hline \multirow[t]{2}{*}{9} & \multirow{2}{*}{\multicolumn{2}{|c|}{ Basis-DRGs F14, F59 }} & Mäßig komplexe Gefäßeingriffe & $\begin{array}{l}\text { Abwertung nichtmehrzeitiger, nichtbeidseitiger Applikation von Stents bei } \\
\text { pAVK }\end{array}$ \\
\hline & & & & Abwertung verschiedener Stent-Kombinationseingriffe \\
\hline
\end{tabular}

setzungen durch fehlende Vergütungsund damit auch seitens der Kostenträger Prüfungsrelevanz kommen wird.

Ein Hauptstreitpunkt lag dabei oft auf der Frage, inwieweit der Revisionscharakter eines Eingriffes bereits durch die Lokalisationskodierung „,künstliches Gefäß“ etc. impliziert sei und eine zusätzliche Kodierung des Revisionskodes dann im Sinne des Grundsatzes der monokausalen Kodierung unterbleiben müsse. Hier hätte Klarheit dadurch geschaffen werden können, dass im OPS-Katalog die notwendigen Voraussetzungen für eine Kodierbarkeit der Revisions-OPS-Kodes aufgenommen worden wären. Insofern stellt die aktuell gefundene Lösung kein wirklich inhaltlich befriedigendes Ergebnis aus Sicht der Leistungserbringer dar. Letztlich haben hier aber vermutlich die Daten des InEK aufgrund der zwischen den Kliniken sehr unterschiedlichen Umsetzbarkeit der Kodierung im Rahmen von Streitigkeiten eine sichere Kostendifferenzierung zwischen den Fällen mit und ohne Revision nicht mehr möglich gemacht.
Neustrukturierung von Zusatzentgelten für patientenindividuelle Aortenprothesen und aortale Stent-Prothesen mit Öffnungen

Wie bereits dargestellt, führte der OPSKode 5-38a.w (Endovaskuläre Implantation von Stent-Prothesen: Patientenindividuell hergestellte Stent-Prothesen) im Vorjahr erstmalig in das Zusatzentgelt ZE2020-53. Das führte dazu, dass bei Implantation einer fenestrierten oder gebranchten Stentgraft-Prothese dieses ZE zumeist mehrfach ausgelöst wurde. Zum einen führten alle Kodes für die Implantation von Stent-Prothesen an thorakaler oder thorakoabdominaler Aorta in dieses ZE, zum anderen wurde es dann noch einmal für die Kodierung der Patientenindividualität abgerechnet. Da nahezu alle dort relevanten Stent-Prothesen patientenindividuell waren und sind, war dieser Zustand für alle Beteiligten unbefriedigend.

Mit dem Jahr 2021 wurde die Bedeutung dieses OPS-Kodes verändert. Nunmehr führen alle dargestellten endovaskulär eingebrachten Stent-Prothesen in das neue ZE2021-189, während der OPSKode 5-38a.w nunmehr nur noch in Verbindung mit einer Stent-Prothese ohne Öffnung an allen drei Abschnitten der
Aorta in das ebenfalls neue ZE2021-188 führt (• Tab. 7; • Abb. 6).

\begin{tabular}{l}
\hline \) Alle dargestellten \\
endovaskulär eingebrachten \\
Stent-Prothesen führen nunmehr \\
in das neue ZE2021-189
\end{tabular}

Die dargestellte Modifikation ist insofern bemerkenswert, als die individuelle Herstellung einer Rohrprothese eher selten sein dürfte. Häufiger wird die bisher nicht mit einem Zusatzentgelt versehene Kombination der Implantation einer StentProthese ohne Öffnungen in Kombination mit einer intraoperativ angefertigten Prothese in der Praxis Relevanz haben.

\section{Abwertung minimal-invasiver Eingriffe an den Herzklappen}

Minimal-invasive Eingriffe an den Herzklappen werden bis auf aktuell noch über NUB-Entgelte finanzierte Verfahren regelhaft in die Basis-DRG F98 (Komplexe minimal-invasive Operationen an Herzklappen ohne minimal-invasiven Eingriff an mehreren Herzklappen) eingruppiert. Unverändert zum Vorjahr erfolgt dabei die Gruppierung transapikaler Eingriffe in die aG-DRG F98A, transfemora- 


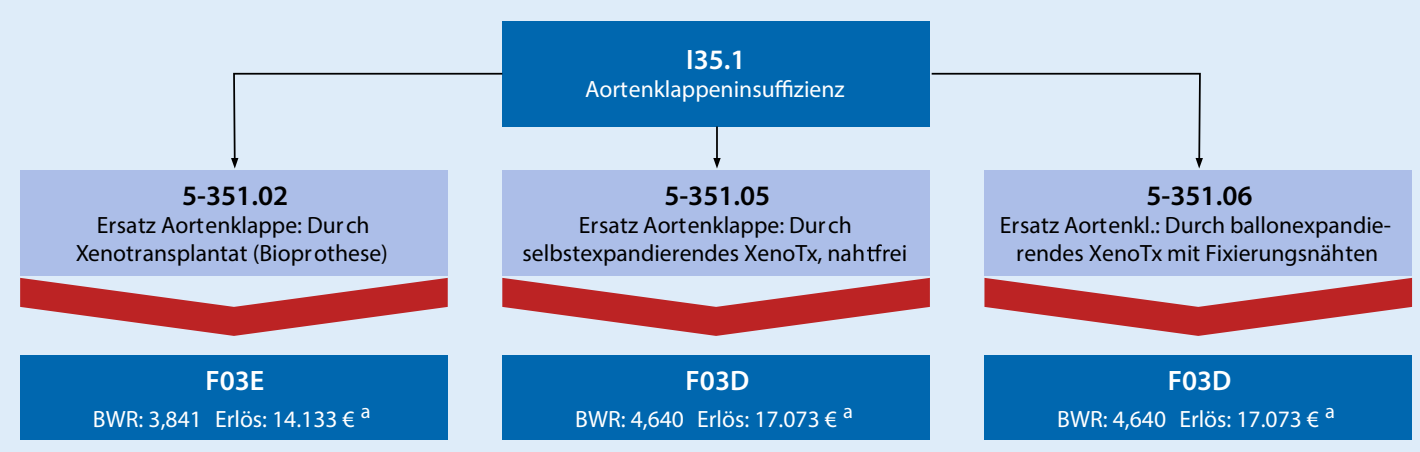

\begin{tabular}{|c|c|c|c|c|c|c|}
\hline & $\begin{array}{c}\text { Venenbypass } \\
\text { einfach }\end{array}$ & $\begin{array}{c}\text { Arterienbypass } \\
\text { einfach }\end{array}$ & $\begin{array}{c}\text { Venenbypass } \\
\text { einfach }\end{array}$ & $\begin{array}{c}\text { Arterienbypass } \\
\text { einfach }\end{array}$ & $\begin{array}{c}\text { Venenbypass } \\
\text { einfach }\end{array}$ & $\begin{array}{c}\text { Arterienbypass } \\
\text { einfach }\end{array}$ \\
\hline 1 fach & \multicolumn{2}{|c|}{ F03D } & F03C & $21.585 \epsilon^{a}$ & $\begin{array}{c}\text { F03B } \\
\text { BWR: } 7,087 \\
\text { Erlös: } 26.077 €^{\mathrm{a}}\end{array}$ & $\begin{array}{c}\text { F03C } \\
\text { BWR: } 5,866 \\
\text { Erlös: } 21.585 €^{\mathrm{a}}\end{array}$ \\
\hline
\end{tabular}

\begin{tabular}{|c|c|c|c|c|c|c|}
\hline & $\begin{array}{c}\text { Venenbypass } \\
\text { zweifach }\end{array}$ & $\begin{array}{l}\text { Arterienbypass } \\
\text { zweifach }\end{array}$ & $\begin{array}{l}\text { Venenbypass } \\
\text { zweifach }\end{array}$ & $\begin{array}{l}\text { Arterienbypass } \\
\text { zweifach }\end{array}$ & $\begin{array}{l}\text { Venenbypass } \\
\text { zweifach }\end{array}$ & $\begin{array}{l}\text { Arterienbypass } \\
\text { zweifach }\end{array}$ \\
\hline $2 \mathrm{fach}$ & \multicolumn{2}{|c|}{ F03D } & \multicolumn{2}{|c|}{ BWR: 5,866 Erlös: $21.585 €$ a } & $\begin{array}{c}\text { F03B (F03A) } \\
\text { BWR: } 7,087(7,534) \\
\text { Erlös: } 26.077 €^{\mathrm{a}}\end{array}$ & $\begin{array}{c}\text { F03C } \\
\text { BWR: } 5,866 \\
\text { Erlös: } 21.585 €^{\mathrm{a}}\end{array}$ \\
\hline
\end{tabular}

\begin{tabular}{|c|c|c|c|c|c|c|}
\hline \multirow{2}{*}{$\begin{array}{c}\text { CAB } \\
3 \text { fach }\end{array}$} & $\begin{array}{c}\text { Venenbypass } \\
\text { dreifach }\end{array}$ & $\begin{array}{c}\text { Arterienbypass } \\
\text { dreifach }\end{array}$ & $\begin{array}{l}\text { Venenbypass } \\
\text { dreifach }\end{array}$ & $\begin{array}{l}\text { Arterienbypass } \\
\text { dreifach }\end{array}$ & $\begin{array}{l}\text { Venenbypass } \\
\text { dreifach }\end{array}$ & $\begin{array}{l}\text { Arterienbypass } \\
\text { dreifach }\end{array}$ \\
\hline & \multicolumn{2}{|c|}{ F03D } & \multicolumn{2}{|c|}{ F03C } & F03B (F03A) & 3A) $26.077 €^{a}$ \\
\hline
\end{tabular}

Abb. 2 \ DRG-Gruppierung unterschiedlicher Klappenprothesen bei Ersatz der Aortenklappen in Kombination mit unter-

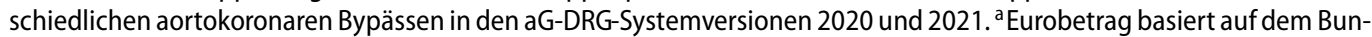
desbasisfallwert 2020. BWR Bewertungsrelation

\begin{tabular}{|c|l|}
\hline Kodes & \multicolumn{1}{c|}{ Text } \\
\hline 135.2 & Aortenklappenstenose mit Insuffizienz \\
$5-384.12$ & Resektion und Ersatz (Interposition) an der Aorta: Aorta ascendens mit \\
& Reimplantation der Koronararterien: Mit Rohrprothese bei Aneurysma \\
& Wechsel von Herzklappenprothesen: Aortenklappe: Xenotransplantat durch \\
$5-352.03$ & Xenotransplantat \\
$5-379.5$ & Andere Operationen an Herz und Perikard: Reoperation \\
\hline
\end{tabular}

\begin{tabular}{|cc|cc|}
\hline DRG & BWR & DRG & BWR \\
F07B & 6,181 & F07A & 6,585 \\
& $22.744 €$ & 5 & \multicolumn{2}{c|}{$24.230 €$} & \\
\hline $\begin{array}{c}\text { 1. Tag Abschlag } \\
\text { Abschlag/Tag }\end{array}$ & $0,452(1663 €)$ & $\begin{array}{c}\text { 1. Tag Abschlag } \\
\text { Abschlag/Tag }\end{array}$ & $0,655(2410 €)$ \\
\hline \multicolumn{2}{|c|}{$1487 €(6,5 \%)$} \\
\hline
\end{tabular}

\begin{tabular}{|c|c|}
\hline F07B & $\begin{array}{l}\text { Andere Eingriffe mit HLM, Alter < } 1 \text { J. od. mit best. kompliz. Konst. od. IntK > - / } 368 \\
\text { /- P., ohne best. komp. Eingr. oder Alter }>0 \text { J., IntK < - / 369/- P., mit and. kompl. } \\
\text { Eingr. mit Reop. an Herz oder Perikard oder mit best. and. kompliz. Konst. }\end{array}$ \\
\hline F07A & $\begin{array}{l}\text { Andere Eingriffe mit Herz-Lungen-Maschine, Alter }<1 \text { Jahr oder mit best. kompliz. } \\
\text { Konstellation oder komp. Operation oder IntK }>-/ 368 / \text { - P. oder Alter }<18 \text { Jahre } \\
\text { mit Reop. Herz od. Perikard oder and. kompliz Konstellation, mit best. kompl. } \\
\text { Eingriffen }\end{array}$ \\
\hline
\end{tabular}

Abb. $3<$ Gruppierungsbeispiel für die Aufwertung des Aszendensersatzes bei Reimplantation der Koronarien. BWR Bewertungsrelation 


\section{Krankenhausmanagement}

\begin{tabular}{|c|c|c|c|}
\hline Kodes & \multicolumn{3}{|c|}{ Text } \\
\hline T82.8 & \multicolumn{3}{|c|}{$\begin{array}{l}\text { Sonstige näher bezeichnete Komplikationen durch Prothesen, Implantate } \\
\text { oder Transplantate im Herzen und in den Gefäßen }\end{array}$} \\
\hline 5-393.54 & \multicolumn{3}{|c|}{$\begin{array}{l}\text { Anlegen eines anderen Shuntes und Bypasses an Blutgefäßen: A. femoralis: } \\
\text { Femoropopliteal, unterhalb des Kniegelenkes }\end{array}$} \\
\hline $5-394.2$ & \multicolumn{3}{|c|}{ Revision einer Blutgefäßoperation: Revision eines vaskulären Implantates } \\
\hline DRG 2020 & BWR & DRG 2021 & BWR \\
\hline F08D & 2,927 & F08E & 2,444 \\
\hline \multicolumn{2}{|c|}{$10.770 €$} & \multicolumn{2}{|c|}{$8.993 €$} \\
\hline 1. Tag Abschlag & 4 & 1. Tag Abschlag & 3 \\
\hline Abschlag/Tag & $0,251(924 €)$ & Abschlag/Tag & $0,238(876 €)$ \\
\hline
\end{tabular}

Rekonstruktive Gefäßeingriffe ohne kompl. Konst., ohne kompl. Aorteneingriff, mit kompl.

F08D Eingr. mit Mehretagen- oder Aorteneingriff oder Reop. oder best. Byp., ohne äuß. schw. CC, ohne best. Aorteneingriff oder bestimmte Bypässe mit äußerst schweren CC

Rekonstruktive Gefäßeingriffe ohne kompl. Konst., ohne kompl. Vakuumbeh., ohne kompl.

F08E Aorteneingriff, mit komplex. Eingriff, ohne Mehretagen- oder Aorteneingriff, ohne Reop., ohne bestimmten Bypass, ohne äußerst schwere CC, ohne bestimmten Aorteneingriff

Abb. $4<$ Beispiel einer Abwertung von Revisionseingriffen in der Basis-DRG F08. BWR Bewertungsrelation

\begin{tabular}{|c|c|c|c|}
\hline Kodes & \multicolumn{3}{|c|}{ Text } \\
\hline 170.23 & \multicolumn{3}{|c|}{$\begin{array}{l}\text { Atherosklerose der Extremitätenarterien: Becken-Bein-Typ, mit } \\
\text { Ruheschmerz }\end{array}$} \\
\hline 5-394.10 & \multicolumn{3}{|c|}{$\begin{array}{l}\text { Revision einer Blutgefäßoperation: Revision einer } \\
\text { Anastomose: Implantat }\end{array}$} \\
\hline DRG & BWR & DRG & BWR \\
\hline F14B & 2,159 & F59C & 1,606 \\
\hline \multicolumn{2}{|c|}{$7.944 €$} & \multicolumn{2}{|c|}{$5.909 €$} \\
\hline $\begin{array}{l}\text { 1. Tag Abschlag } \\
\text { Abschlag/Tag }\end{array}$ & $\begin{array}{c}3 \\
0,254(935 €)\end{array}$ & $\begin{array}{l}\text { 1. Tag Abschlag } \\
\text { Abschlag/Tag }\end{array}$ & $\begin{array}{c}1 \\
0,404(1487 €)\end{array}$ \\
\hline \multicolumn{4}{|c|}{$-2035 €(-25,6 \%)$} \\
\hline F14B & \multicolumn{3}{|c|}{$\begin{array}{l}\text { Komplexe oder mehrfache Gefäßeingriffe außer große } \\
\text { rekonstruktive Eingriffe, ohne äußerst schwere CC }\end{array}$} \\
\hline F59C & \multicolumn{3}{|c|}{$\begin{array}{l}\text { Mäßig komplexe Gefäßeingriffe ohne äußerst schwere CC, ohne } \\
\text { aufwendige Gefäßintervention, mit aufwendigem Eingriff oder } \\
\text { Mehrfacheingriff oder bestimmter Diagnose oder Alter }<16 \\
\text { Jahre, mehr als ein Belegungstag }\end{array}$} \\
\hline
\end{tabular}

\begin{tabular}{|c|c|c|c|}
\hline Kodes & \multicolumn{3}{|c|}{ Text } \\
\hline 170.25 & \multicolumn{3}{|c|}{$\begin{array}{l}\text { Atherosklerose der Extremitätenarterien: Becken-Bein-Typ, mit } \\
\text { Gangrän }\end{array}$} \\
\hline $5-381.70$ & \multicolumn{3}{|c|}{ Endarteriektomie: Arterien Oberschenkel: A. femoralis } \\
\hline $5-394.10$ & \multicolumn{3}{|c|}{$\begin{array}{l}\text { Revision einer Blutgefäßoperation: Revision einer Anastomose } \\
\text { Implantat }\end{array}$} \\
\hline DRG & BWR & DRG & BWR \\
\hline F14B & 2,159 & F14B & 2,241 \\
\hline \multicolumn{2}{|c|}{$7.944 €$} & \multicolumn{2}{|c|}{$8.246 €$} \\
\hline 1. Tag Abschlag & 3 & 1. Tag Abschlag & 3 \\
\hline Abschlag/Tag & $0,254(935 €)$ & Abschlag/Tag & $0,263(968 €)$ \\
\hline \multicolumn{4}{|c|}{$302 €(3,8 \%)$} \\
\hline F14B & \multicolumn{3}{|c|}{$\begin{array}{l}\text { Komplexe oder mehrfache Gefäßeingriffe außer große } \\
\text { rekonstruktive Eingriffe, ohne äußerst schwere CC }\end{array}$} \\
\hline F14B & \multicolumn{3}{|c|}{$\begin{array}{l}\text { Komplexe oder mehrfache Gefäßeingriffe außer große } \\
\text { rekonstruktive Eingriffe, ohne äußerst schwere CC }\end{array}$} \\
\hline
\end{tabular}

Hier ist das pAVK-Stadium gruppierungsrelevant!

Abb. 5 - Veränderungen der Gruppierungsrelevanz von Revisions-OPS-Kodes an Blutgefäßen für die Basis-DRGF14 Komplexe und mehrfache Gefäßeingriffe in den aG-DRG-Systemversionen 2020 und 2021 in Abhängigkeit des Stadiums der peripheren arteriellen Verschlusskrankheit ( $p A V K)$. BWR Bewertungsrelation 
Tab. 6 Gruppierungsrelevanz von Revisions-OPS-Kodes an Blutgefäßen für die Basis-DRGF14

Komplexe und mehrfache Gefäßeingriffe in den aG-DRG-Systemversionen 2020 und 2021 in Ver-

bindung mit weniger komplexen Stadien der PAVK

\begin{tabular}{|c|c|c|c|}
\hline \multirow[t]{2}{*}{$\begin{array}{l}\text { OPS- } \\
\text { Kode }\end{array}$} & \multirow[t]{2}{*}{ OPS-Text } & \multicolumn{2}{|c|}{$\begin{array}{l}\text { Zuordnung zur Basis } \\
\text { DRG F14 }\end{array}$} \\
\hline & & Bis 2020 & Ab 2021 \\
\hline $5-394.0$ & $\begin{array}{l}\text { Revision einer Blutgefäßoperation: Operative Behandlung } \\
\text { einer Blutung nach Gefäßoperation }\end{array}$ & $\mathrm{X}$ & $\mathrm{X}$ \\
\hline $5-394.1$ & Revision einer Blutgefäßop.: Revision einer Anastomose & $x$ & - \\
\hline $5-394.2$ & $\begin{array}{l}\text { Revision einer Blutgefäßop.: Revision eines vaskulären Im- } \\
\text { plantates }\end{array}$ & $\mathrm{x}$ & - \\
\hline $5-394.3$ & $\begin{array}{l}\text { Revision einer Blutgefäßop.: Wechsel eines vaskulären Im- } \\
\text { plantates }\end{array}$ & $x$ & $x$ \\
\hline
\end{tabular}

Tab. 7 Umstrukturierung des Zusatzentgeltes (ZE) für aortale Stentgrafts

\begin{tabular}{|c|c|c|c|c|c|}
\hline ZE 2021 & ZE-Text & OPS & Endovaskuläre Implantation von Stent-Prothesen: & ZE2020-53 & Neues ZE \\
\hline \multirow[t]{4}{*}{ ZE2021-188 } & \multirow{4}{*}{$\begin{array}{l}\text { Patientenindividuell her- } \\
\text { gestellte Stent-Prothesen } \\
\text { an der Aorta, ohne Öff- } \\
\text { nung }\end{array}$} & 5-38a.70 & Aorta thoracica: Stent-Prothese, ohne Öffnung & - & $x$ \\
\hline & & $5-38 a .80$ & Aorta thoracoabdominalis: Stent-Prothese, ohne Öffnung & - & $\mathrm{x}$ \\
\hline & & 5-38a.c0 & Aorta abdominalis: Stent-Prothese, ohne Öffnung & - & $\mathrm{x}$ \\
\hline & & $+5-38 a . w$ & Patientenindividuell hergestellte Stent-Prothesen & $\mathrm{X}$ & - \\
\hline \multirow[t]{10}{*}{ ZE2021-189 } & \multirow{10}{*}{$\begin{array}{l}\text { Stent-Prothesen an der } \\
\text { Aorta, mit Öffnung }\end{array}$} & $5-38 a .7 b$ & Aorta thoracica: Stent-Prothese, mit 1 Öffnung & $\mathrm{X}$ & - \\
\hline & & $5-38 a .7 c$ & ... mit 2 Öffnungen & $x$ & - \\
\hline & & $5-38 a .7 d$ & ... mit 3 oder mehr Öffnungen & $x$ & - \\
\hline & & $5-38 a .8 c$ & Aorta thoracoabdominalis: Stent-Prothese, mit 1 Öffnung & $x$ & - \\
\hline & & $5-38 a .8 d$ & ... mit 2 Öffnungen & $x$ & - \\
\hline & & $5-38 a .8 e$ & ... mit 3 Öffnungen & $x$ & - \\
\hline & & $5-38 a .8 f$ & ... mit 4 oder mehr Öffnungen & $x$ & - \\
\hline & & 5-38a.c1 & Aorta abdominalis: Stent-Prothese, mit 1 Öffnung & $x$ & - \\
\hline & & $5-38 a \cdot c 2$ & ... mit 2 Öffnungen & $\mathrm{x}$ & - \\
\hline & & $5-38 a . c 3$ & ... mit 3 oder mehr Öffnungen & $x$ & - \\
\hline
\end{tabular}

ler Eingriffe (z. B. Transkatheter-Aortenklappenimplantation, TAVI) in die F98B und rekonstruktiver Eingriffe (z. B. Clipping an der Mitral- oder Trikuspidalklappe, Spangenrekonstruktionen über den Koronarsinus) in die F98C. Für diese aGDRG lassen sich relevante Abwertungen für das Jahr 2021 finden. Diese führen in vielen interventionellen herzchirurgischen Abteilungen zu erheblichen negativen Katalogeffekten (• Abb. 7).

\section{Zusammenfassung und Fazit für die Praxis}

Während in vielen Fachgebieten nur geringe G-DRG-Systemumbauten für das Jahr $2021 \mathrm{zu}$ finden sind - exemplarisch sei hier auf das Fach der Kardiologie verwiesen - ist die Herz- und Gefäßchirurgie vielfältig von z.T. erheblichen Änderungen mit Auswirkungen auf die Erlöse betroffen. Wie in den vergangenen Jah- ren auch, werden viele der Änderungen in der aG-DRG-Gruppierung sehr unterschiedlich in den einzelnen Kliniken in Abhängigkeit vom Fallspektrum wirken. Neben den dargestellten geänderten Kodierungen und den veränderten Gruppierungsalgorithmen kommt gerade für das Jahr 2021 auch wieder die Abwertung vieler ICD-Kodes in Nebendiagnoseposition für die Ermittlung des PCCL-Wertes zum Tragen. Der PCCL-Wert ist eine Kennzahl für die Komorbidität der Patienten und kann je nach Ausprägung auch erlösrelevant sein. Insbesondere in der Gefäßmedizin hat der PCCL-Wert auch weiterhin einen erheblichen Einfluss auf den aG-DRG-Erlös. Die Abwertung von nahezu 2000 verschiedenen ICD-Kodes (als Nebendiagnosen kodiert) in unterschiedlichen Konstellationen für das Jahr 2021 führt in den Katalogeffekten entsprechend ausgerichteter Kliniken bzw. Fachabteilungen somit wesentlich zu Ab- wertungen. Der Katalogeffekt beschreibt die unterschiedliche Bewertung derselben Leistungen in zwei verschiedenen DRG-Versionen.

Ein weiterer, immer dringlicherer die Gefäßmedizin betreffender Aspekt ist der zunehmende Druck zur Ambulantisierung von Leistungen. Hier wird der im Jahre 2022 erwartete und voraussichtlich deutlich erweiterte AOP-Katalog noch einmal eine deutliche Dynamik zur Ambulantisierung bisher noch stationär erbrachter Leistungen auslösen, insbesondere in der Gefäßchirurgie. Daher sollten in jeder Klinik die Strukturen für die Durchführung ambulanter Operationen überdacht und Prozesse neu aufgesetzt werden, um die zunehmende Anzahl möglicherweise stationär nicht mehr vergütungsfähiger Eingriffe trotzdem im Sinne einer vollständigen Aus- und Weiterbildung von Personal und auch im Sinne einer umfänglichen Versorgungs- 
Endovaskuläre Implantation von Stent-Prothesen:

5-38a.70

Aorta thoracica: Stent-Prothese, ohne Öffnung

5-38a.80

Aorta thoracoabdominalis: Stent-Prothese, ohne Öffnung

5-38a.c0

Aorta abdominalis: Stent-Prothese, ohne Öffnung

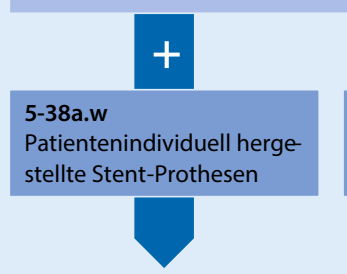

ZE2021-188

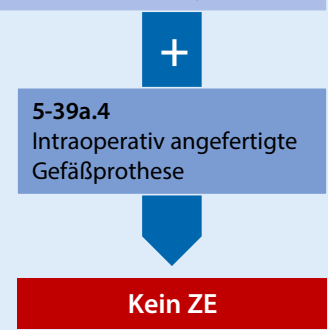

Endovaskuläre Implantation von Stent-Prothesen: 5-38a.7- (.7b bis .7d)

Aorta thoracica: Stent-Prothese, mit Öffnungen

5-38a.8- (.8c bis .8f)

Aorta thoracoabdominalis: Stent-Prothese, mit Öffnungen

5-38a.c- (.c1 bis .c3)

Aorta abdominalis: Stent-Prothese, mit Öffnungen

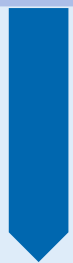

ZE2021-189
Abb. $6<$ Neustrukturierung der Zusatzentgelte (ZE) für patientenindividuell hergestellte StentProthesen an der Aorta ohne Öffnung (ZE2021-188) und Stent-Prothesen an der Aorta mit Öffnung (ZE2021189)

\begin{tabular}{|c|c|c|c|c|c|c|}
\hline DRG & Bezeichnung & $\begin{array}{c}\text { CW } \\
2020\end{array}$ & $\begin{array}{r}\text { CW } \\
2021\end{array}$ & & $\begin{array}{l}\text { Diff } \\
\text { CW }\end{array}$ & $\begin{array}{r}\text { Diff } \\
\text { CW\% }\end{array}$ \\
\hline F98A & $\begin{array}{l}\text { Komplexe minimal-invasive Operationen an Herzklappen } \\
\text { ohne minimal-invasiven Eingriff an mehreren Herzklappen, } \\
\text { mit hochkomplexem Eingriff oder komplexer Diagnose } \\
\text { oder Alter < } 30 \text { Jahre oder Implantation eines Wachstums- } \\
\text { stents }\end{array}$ & 7,729 & 7,296 & $\rightarrow$ & $-0,433$ & $-6 \%$ \\
\hline F98B & $\begin{array}{l}\text { Komplexe minimal-invasive Operationen an Herzklappen } \\
\text { ohne minimal-invasiven Eingriff an mehreren Herzklappen, } \\
\text { ohne hochkomplexen Eingriff, ohne komplexe Diagnose, } \\
\text { Alter > } 29 \text { Jahre, ohne Implantation eines Wachstums- } \\
\text { Stents, mit sehr komplexem Eingriff }\end{array}$ & 6,694 & 6,173 & $\rightarrow$ & $-0,521$ & $-8 \%$ \\
\hline F98C & $\begin{array}{l}\text { Komplexe minimal-invasive Operationen an Herzklappen } \\
\text { ohne minimal-invasiven Eingriff an mehreren Herzklappen, } \\
\text { ohne hochkomplexen Eingriff, ohne komplexe Diagnose, } \\
\text { Alter > } 29 \text { Jahre, ohne Implantation eines Wachstums- } \\
\text { stents, ohne sehr komplexen Eingriff }\end{array}$ & 8,607 & 8,238 & $\rightarrow$ & $-0,369$ & $-4 \%$ \\
\hline
\end{tabular}

Abb. $7 \triangleleft$ Abwertungen in der Basis-DRG F98. CW "Cost weight"/ Bewertungsrelation möglichkeit der Patienten wirtschaftlich tragfähig erbringen zu können.

Insofern wird auch das Jahr 2021, völlig abgesehen vom weiteren Verlauf der aktuellen Pandemie, weiterhin eine intensive Beschäftigung mit der Abrechnungsrealität und der Entwicklung zukunftsfähiger Szenarien erfordern.

\section{Korrespondenzadresse}

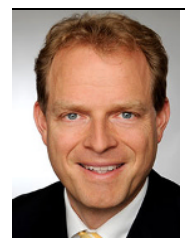

PD Dr. Dominik Franz

Franz und Wenke - Beratung

im Gesundheitswesen GbR

Mendelstr. 11, 48149 Münster,

Deutschland

d.franz@

dasgesundheitswesen.de
PD Dr. Dominik Franz Berufsausbildung zum Bankkaufmann, danach Studium der Humanmedizin an der Universität Hamburg und der Medizinischen Hochschule Hannover. Klinische Tätigkeit an der Universitäts-Hals-Nasen-Ohren-Klinik Freiburg i. Br., danach Wechsel in die Stabsstelle Medizincontrolling des Universitätsklinikums Münster (UKM). Seither Mitglied der DRG-Research-Group. Erlangung der Zusatzbezeichnung "Medizinische Informatik". Habilitation zum Thema "Krankenhausmanagement" im Jahre 2014. Im Jahre 2016 stellv. Leiter des Geschäftsbereichs Medizinisches Management des UKM. Seit 2017 Gründer und geschäftsführender Gesellschafter der Franz und Wenke - Beratung im Gesundheitswesen GbRin Münster.

\section{Einhaltung ethischer Richtlinien}

Interessenkonflikt. D. Franz, A. Wenke und N. Roeder geben an, dass kein Interessenkonflikt besteht.
Für diesen Beitrag wurden von den Autoren keine Studien an Menschen oder Tieren durchgeführt. Für die aufgeführten Studien gelten die jeweils dort angegebenen ethischen Richtlinien.

\section{Literatur}

1. Heilemann K, Schlottmann N (2021) G-DRGSystem und Kodierrichtlinien (DKR) 2021. Krankenhaus 1:21-36

2. Institut für das Entgeltsystem im Krankenhaus gGmbH (2020) Fallpauschalen-Katalog 2021 (www.g-drg.de)

3. Institut für das Entgeltsystem im Krankenhaus gGmbH (2020) Abschlussbericht Weiterentwicklung des aG-DRG-Systems für das Jahr 2021 (www. g-drg.de)

4. Institut für das Entgeltsystem im Krankenhaus gGmbH (2020) Deutsche Kodierrichtlinien 2021 (www.g-drg.de) 
5. BfArM (2020) Operationen- und Prozedurenschlüssel (OPS) Version 2021 (www.bfarm.de)

6. BfArM (2020) ICD-10-GM Version 2021 (www. bfarm.de)

7. Institut für das Entgeltsystem im Krankenhaus gGmbH (2020) aG-DRG-Reportbrowser 2021 (www.g-drg.de)

8. Institut für das Entgeltsystem im Krankenhaus gGmbH (2019) aG-DRG-Reportbrowser 2020 (www.g-drg.de)

Debus, E. Sebastian, Gross-Fengels, Walter (Hrsg.)

\section{Operative und interventionelle Gefäßmedizin}

Berlin Heidelberg: Springer-Verlag 2020, 2. Auflage, 1276 S., 530 Abb., (ISBN: 9783-662-53378-9), 299 EUR

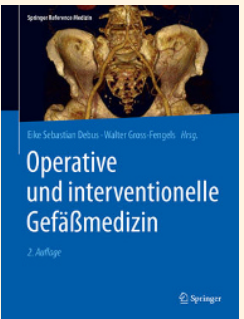

Das zweibändige Lehrbuch "Operative und interventionelle Gefäßmedizin", herausgegeben von Eike Sebastian Debus und Walter Gross-Fengels im Springerverlag,

ist in seiner Neuauflage ein Werk, dass in deutscher Sprache das breite Spektrum der vaskulären Medizin umfassend abdeckt. Bereits mit der ersten Auflage avancierte dieses Lehrbuch in den letzten 9 Jahren zu einem, zu dem Standardwerk im deutschsprachigen Raum.

In der nun 2. komplett überarbeiteten und aktualisierten Auflage finden sich erweiterte und neue Kapitel, die der schnellen Entwicklung und Innovationen des Faches Gefäßmedizin gerecht werden.

Dieses Lehrbuch, das von unterschiedlichen sehr kompetenten Autoren geschrieben wurde, liest sich für mich nicht nur flüssig und stimmig, sondern spannend. Trotz der schnell zunehmenden Evidenz, die v.a. in den digitalen Medien abrufbar ist und mit der Printmedien kaum mithalten können, ist dieses Standardwerk sehr aktuell.

Das Lehrbuch ist sehr gut strukturiert, so dass man schnell eine Antwort auf die aktuelle Frage erhält. Die zum Teil komplexen Krankheitsbilder werden sehr gut dargestellt und wenn nötig miteinander verknüpft. Die multimodalen Therapieansätze (konservativ, endovaskulär und offene Chirurgie) finden dabei in den gesonderten Kapiteln in Theorie und Praxis eine besondere und differenzierte Beachtung. Die Tatsache, dass beide Herausgeber sowohl Gefäßchirurg als auch interventioneller Radiologe sind, und über eine exzellente jahrelange klinische und wissenschaftliche Erfahrung verfügen, stellen meines Erachtens die entscheidende
Grundlage dafür dar, dass eine ausgewogene und zugleich differenzierte Darstellung der Gefäßmedizin in Klinik und Praxis in beiden Bänden nachzulesen ist.

Mein Fazit: Das Lehrbuch „Operative und interventionelle Gefäßmedizin“ bereichert als Lehrbuch nicht nur die persönliche Bibliothek des Assistenten in der Weiterbildung, sondern stellt auch ein umfangreiches, interessantes Nachschlagewerk für den fortgeschrittenen Facharzt dar. Es deckt inhaltlich das Curriculum der Weiterbildung in der Gefäßchirurgie ab.

Einzigartig wird dieses Werk durch seine multidisziplinäre sehr kompetente Autorenschaft.

Es findet meine uneingeschränkte Empfehlung und sollte in lihrer Bibliothek nicht fehlen, zumal das Preis Leistungsverhältnis absolut stimmig ist!

D. Böckler (Heidelberg) 
Hier steht eine Anzeige.

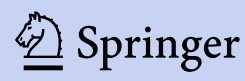

